\title{
Nonlinear Phase Noise Estimate Based on Electronic Orthogonal Coherent for 112 Gb/s PDM-4QAM System
}

\author{
Baocun Wang1, Bin Li1,2*, Fengguang Luo', Benxiong Huang',3, Ming Tian', Xuecheng Zou' \\ ${ }^{1}$ Institute of Optics and Electronic Imformation, Huazhong University of Science and Technology, Wuhan, China \\ ${ }^{2}$ School of Electronic and Imformation Engineering, Huazhong University of Science and Technology, Wuhan, \\ China \\ ${ }^{3}$ National Engineering Laboratory for Next Generation Internet Acess System, Wuhan, China \\ Email: ${ }^{*}$ libin8208@126.com
}

Received 16 September 2014; revised 12 October 2014; accepted 8 November 2014

Copyright (C) 2014 by authors and Scientific Research Publishing Inc.

This work is licensed under the Creative Commons Attribution International License (CC BY).

http://creativecommons.org/licenses/by/4.0/

c) (7) Open Access

\begin{abstract}
In this paper, we proposed a novel method of joint phase noise estimate (JPNE) for PDM-M-QAM $(M=4,16,32,64, \ldots)$ transmission systems, and established the theoretical model to illustrate the operation mechanism. The simulation of laser phase noise and fiber nonlinearity compensation based on the proposed JPNE method had also been demonstrated. For 112 Gb/s PDM-4QAM transmission system, the simulation results had showed that the optimum launch power increased from $-4 \mathrm{dBm}$ to at least $0 \mathrm{dBm}$ compared with the condition of no phase noise compensation in reach of all simulation distances.
\end{abstract}

\section{Keywords}

Joint Phase Noise Estimate, Fiber Nonlinearity, PDM-4QAM, Optimum Launch Power, Phase Noise Compensation

\section{Introduction}

Recently, long haul fiber optic transmission based on quadrature amplitude modulation (QAM) has drawn significant research interest [1]. Coherent detection together with the polarization division multiplexing (PDM-) QAM format has paved the way for robust transmission systems at $100 \mathrm{~Gb} / \mathrm{s}$ or beyond. Indeed, these solutions benefit from the capacity of advanced digital signal processing (DSP) to compensate for linear impairments,

\footnotetext{
${ }^{*}$ Corresponding author.
}

How to cite this paper: Wang, B.C., Li, B., Luo, F.G., Huang, B.X., Tian, M. and Zou, X.C. (2014) Nonlinear Phase Noise Estimate Based on Electronic Orthogonal Coherent for 112 Gb/s PDM-4QAM System. Optics and Photonics Journal, 4, 316-324. http://dx.doi.org/10.4236/opj.2014.411032 
which made the transmission system remarkable tolerance to chromatic dispersion (CD) and polarization mode dispersion (PMD). In addition, laser phase noise (LPN) can also be mitigated by feed forward carrier recovery (FFCR) techniques with penalties depending on the transmitter (TX) and receiver (RX) laser linewidth-to-symbol rate ratio, however, the computation complexity of FFCR scheme increases significantly for higher-order modulation formats (M-QAM, $M=16,32,64, \ldots$ ) [2]. However, nonlinear (NL) impairments caused by fiber Kerreffect are still a major problem in long-haul transmission systems since it limits the maximum launch power, which in turn reduces the maximum achievable reach. Recent years, some researchers have deeply studied on compensating both laser phase noise and nonlinear impairments. Sergejs Makovejs et al. proposed a novel digital signal processing technique for carrier phase estimation and symbol estimation in PDM-16QAM and QPSK transmission system; the obtained results demonstrated that the optimum launch power increased from $-4 \mathrm{dBm}$ to $-1 \mathrm{dBm}$ with a consequent increase in maximum reach from $1440 \mathrm{~km}$ to $2400 \mathrm{~km}$ [3] [4]. Mohamed MorsyOsman et al. proposed and experimentally demonstrated polarization demultiplexing, frequency offset and phase noise compensation using an electrically inserted pilot for Single-Carrier Systems, and reported excellent backto-back and transmission performances [5]-[8]. The powerful DSP capacity of coherent receiver can also be used to deal with nonlinear impairments, for example, digital inverse backward-propagation [9]-[12]. However, due to the heavy computation during algorithm processing, it's found that the digital inverse backward-propagation compensation for nonlinear effects was very challenging.

In this paper, we proposed a novel monitoring and compensation method for joint phase noise which included LPN and NL phase disturbance. In transmitter, a RF pilot was inserted into monitored channel, due to that it was affected by LPN and NL as same as in-band signals during transmitting. So we can extract the phase deviation of the received RF pilot in receiver and compensate the receiving signal's phase error based on phase rotation. The theoretical analysis and experimental results were presented. It shows that the proposed method has a significant effect on compensation of joint phase noise in $112 \mathrm{~Gb} / \mathrm{s}$ PDM-4QAM system.

\section{Principle of Joint Phase Deviation Estimate}

The proposed model of joint phase deviation estimate is shown in Figure 1. Two-way orthogonal light were separated by the polarization diversity hybrid, at the X-polarization, the light was separated into two ways by the $90^{\circ}$ phase shift, two ways light were detected by the PD and were orthogonal mixed respectively with the local $\mathrm{RF}$, after, four ways mixed signals respectively get through LPF to remove the high frequency component. The

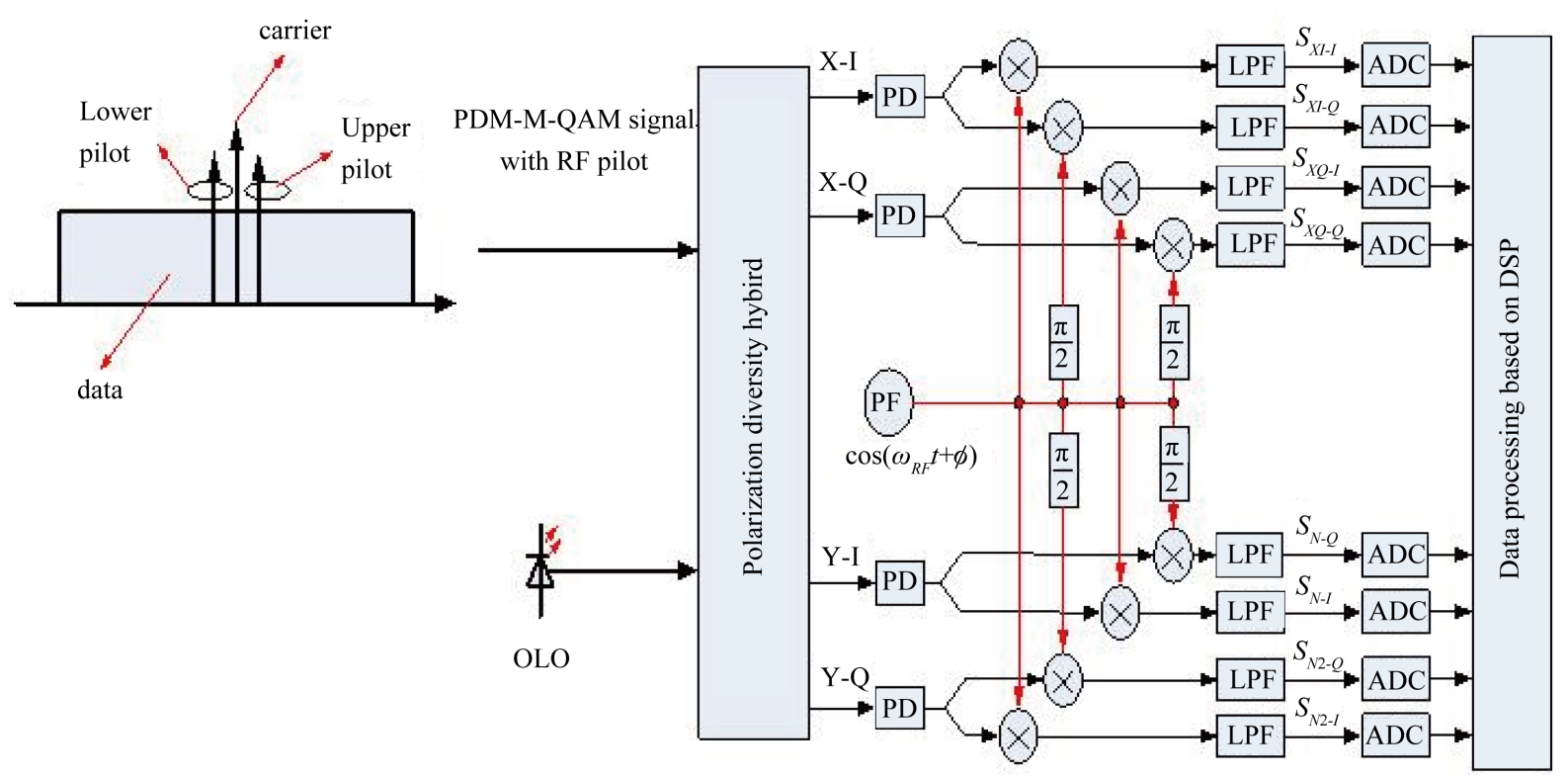

$\begin{array}{llll}\text { Electronic Mixer } & \text { PD Photo-detector LPF Low Pass Filter } \quad \text { ADC Analog to Digital Converter }\end{array}$ 
outputs of LPFs were digital sampled by ADCs and feed into DSP to process based on certain algorithm, and this algorithm was expressed in this paper.

At the Y-polarization, the operating process was same as the X-polarization's. Finally, both of the X-polarization and the Y-polarization signal phase deviation can be estimated respectively.

Following, we illustrate the operation process on X-polarization. The carrier of X-polarization can be given by:

$$
C(t)=\alpha \sqrt{I_{0}} \cos \left(\omega_{0}+\varphi_{0}\right)
$$

and the lower and upper sideband of the pilot at in-phase can be respectively given by:

$$
\begin{aligned}
& E_{\mathrm{IL}}(t)=\beta \sqrt{I_{0}} \cos \left(\left(\omega_{0}-\omega_{d}\right)+\varphi_{\mathrm{IL}}\right) \\
& E_{\mathrm{IU}}(t)=\beta \sqrt{I_{0}} \cos \left(\left(\omega_{0}+\omega_{d}\right)+\varphi_{\mathrm{IU}}\right)
\end{aligned}
$$

and the lower and upper sideband of the pilot at quadrature-phase can be respectively given by:

$$
\begin{aligned}
& E_{\mathrm{QL}}(t)=\beta \sqrt{I_{0}} \cos \left(\left(\omega_{0}+\omega_{d}\right)+\varphi_{\mathrm{QL}}\right) \\
& E_{\mathrm{QU}}(t)=\beta \sqrt{I_{0}} \cos \left(\left(\omega_{0}+\omega_{d}\right)+\varphi_{\mathrm{QU}}\right)
\end{aligned}
$$

where $\omega_{0}$ was the optical carrier frequency, $\varphi_{0}$ was instantaneous phase of the carrier, $\omega_{d}$ was the frequency of the RF pilot, both $\omega_{0}-\omega_{d}$ and $\omega_{0}+\omega_{d}$ were two frequency elements of the lower and upper sideband, $\varphi_{\mathrm{IL}}$ and $\varphi_{\mathrm{IU}}$ were instantaneous phase of $E_{\mathrm{IL}}(t)$ and $E_{\mathrm{IU}}(t)$ respectively, $\varphi_{\mathrm{QL}}$ and $\varphi_{\mathrm{QU}}$ were instantaneous phase of $E_{\mathrm{QL}}(t)$ and $E_{\mathrm{QU}}(t)$ respectively.

During the transmission, the phase of RF pilot must be affected by the fiber nonlinear which contains of cross-phase modulation (XPM), self-phase modulation (SPM), besides, fiber linear impairments (some as chromatic dispersion, polarization mode dispersion) also can load some phase errors on the pilot. So we can estimate the joint phase deviation of the RF pilot to compensate M-QAM signals.

At the receiver, the received $E_{\mathrm{IL}}^{\prime}(t), E_{\mathrm{IU}}^{\prime}(t), E_{\mathrm{QL}}^{\prime}(t), E_{\mathrm{QU}}^{\prime}(t)$ can be respectively given by:

$$
\begin{aligned}
& E_{\mathrm{IL}}^{\prime}(t)=\beta \sqrt{I_{0}} \cos \left(\left(\omega_{0}+\omega_{d}\right)+\varphi_{\mathrm{IL}}+\varphi_{\mathrm{ILCD}}+\varphi_{\mathrm{ILNL}}\right) \\
& E_{\mathrm{IU}}^{\prime}(t)=\beta \sqrt{I_{0}} \cos \left(\left(\omega_{0}+\omega_{d}\right)+\varphi_{\mathrm{IU}}+\varphi_{\mathrm{IUCD}}+\varphi_{\mathrm{IUNL}}\right) \\
& E_{\mathrm{QL}}^{\prime}(t)=\beta \sqrt{I_{0}} \cos \left(\left(\omega_{0}+\omega_{d}\right)+\varphi_{\mathrm{QL}}+\varphi_{\mathrm{QLCD}}+\varphi_{\mathrm{QLNL}}\right) \\
& E_{\mathrm{QU}}^{\prime}(t)=\beta \sqrt{I_{0}} \cos \left(\left(\omega_{0}+\omega_{d}\right)+\varphi_{\mathrm{QU}}+\varphi_{\mathrm{QUCD}}+\varphi_{\mathrm{QUNL}}\right)
\end{aligned}
$$

where $\varphi_{\mathrm{ILCD}}, \varphi_{\mathrm{IUCD}}, \varphi_{\mathrm{QLCD}}, \varphi_{\mathrm{QUCD}}$ were, lower and upper sideband's phase delays respectively that generated by the CD, $\varphi_{\mathrm{ILNL}}, \varphi_{\mathrm{IUNL}}, \varphi_{\mathrm{QLNL}}, \varphi_{\mathrm{QUNL}}$ were lower and upper sideband's phase disturbance respectively that generated by nonlinear effect.

The proposed architecture of joint phase estimate for PDM-M-QAM system is shown in Figure 1. As similar with coherent receiver, a coherent front-end integrates polarization beams splitters (PBSs), an optical hybrid, a local laser and balanced photodetectors (BPDs) to provide four signals corresponding to the $I$ and $Q$ components on both polarizations. After the square-law photodetectors and the electronic mixing, four outputs of low-pass filters (LPF) were given by:

$$
\begin{aligned}
& S_{X I_{-} I}(t)=\sqrt{\left|E_{\mathrm{IU}}^{\prime}(t)+\mathrm{LO}(t)\right|^{2}+\left|E_{\mathrm{IL}}^{\prime}(t)+\mathrm{LO}(t)\right|^{2}} \times H_{I}(t) \\
& S_{X I_{-} Q}(t)=\sqrt{\left|E_{\mathrm{IU}}^{\prime}(t)+\mathrm{LO}(t)\right|^{2}+\left|E_{\mathrm{IL}}^{\prime}(t)+\mathrm{LO}(t)\right|^{2}} \times H_{Q}(t) \\
& S_{X Q_{-} I}(t)=\sqrt{\left|E_{\mathrm{QU}}^{\prime}(t)+\mathrm{LO}(t)\right|^{2}+\left|E_{\mathrm{QL}}^{\prime}(t)+\mathrm{LO}(t)\right|^{2}} \times H_{I}(t) \\
& S_{X Q_{-} Q}(t)=\sqrt{\left|E_{\mathrm{QU}}^{\prime}(t)+\mathrm{LO}(t)\right|^{2}+\left|E_{\mathrm{QL}}^{\prime}(t)+\mathrm{LO}(t)\right|^{2}} \times H_{Q}(t)
\end{aligned}
$$


where $\mathrm{LO}(t)$ was the local optical for coherent receiving, $H_{I}(t), H_{Q}(t)$ were the mixer transfer function which generated by the electronic local oscillator (ELO), $\mathrm{LO}(t), H_{I}(t)$ and $H_{Q}(t)$ were respectively given by:

$$
\begin{aligned}
& \mathrm{LO}(t)=\gamma \sqrt{I_{0}} \cos \left(\omega_{0} t+\varphi_{\mathrm{OLO}}\right) \\
& H_{I}(t)=\cos \left(\omega_{\mathrm{LO}} t+\varphi_{\mathrm{ELO}}\right) \\
& H_{Q}(t)=\cos \left(\omega_{\mathrm{LO}} t+\varphi_{\mathrm{ELO}}+\frac{\pi}{2}\right)
\end{aligned}
$$

where $\omega_{\mathrm{LO}}, \varphi_{\mathrm{ELO}}$ were frequency and instantaneous phase of the electronic local oscillator respectively. When $\omega_{\mathrm{LO}}=\omega_{d}, S_{X I_{-} I}, S_{X I_{-} Q}, S_{X Q_{-} I}, S_{X Q_{-} Q}$ after LPFs can be determined:

$$
\begin{aligned}
& S_{X I_{-} I}(t)=\frac{\alpha \beta I_{0}}{2} \cos \left(\varphi_{\mathrm{ELO}}+2 \varphi_{\mathrm{OLO}}+\varphi_{\mathrm{IL}}+\varphi_{\mathrm{IU}}+\varphi_{\mathrm{ILCD}}+\varphi_{\mathrm{IUCD}}+\varphi_{\mathrm{ILNL}}+\varphi_{\mathrm{IUNL}}\right) \\
& S_{X I_{-} Q}(t)=\frac{\alpha \beta I_{0}}{2} \cos \left(\frac{\pi}{2}+\varphi_{\mathrm{ELO}}+2 \varphi_{\mathrm{OLO}}+\varphi_{\mathrm{IL}}+\varphi_{\mathrm{IU}}+\varphi_{\mathrm{ILCD}}+\varphi_{\mathrm{IUCD}}+\varphi_{\mathrm{ILNL}}+\varphi_{\mathrm{IUNL}}\right) \\
& S_{X Q_{-} I}(t)=\frac{\alpha \beta I_{0}}{2} \cos \left(\varphi_{\mathrm{ELO}}+2 \varphi_{\mathrm{OLO}}+\varphi_{\mathrm{QL}}+\varphi_{\mathrm{QU}}+\varphi_{\mathrm{QLCD}}+\varphi_{\mathrm{QUCD}}+\varphi_{\mathrm{QLNL}}+\varphi_{\mathrm{QUNL}}\right) \\
& S_{X Q_{-} Q}(t)=\frac{\alpha \beta I_{0}}{2} \cos \left(\frac{\pi}{2}+\varphi_{\mathrm{ELO}}+2 \varphi_{\mathrm{OLO}}+\varphi_{\mathrm{QL}}+\varphi_{\mathrm{QU}}+\varphi_{\mathrm{QLCD}}+\varphi_{\mathrm{QUCD}}+\varphi_{\mathrm{QLNL}}+\varphi_{\mathrm{QUNL}}\right)
\end{aligned}
$$

the phase mode values (PMVs) of in-phase and quadrature-phase can respectively be given by:

$$
\begin{aligned}
& \operatorname{PMV}_{I}=\arctan \frac{S_{X I_{-} Q}(t)}{S_{X I_{-} I}(t)} \\
& \operatorname{PMV}_{Q}=\arctan \frac{S_{X Q_{-} Q}(t)}{S_{X Q_{-} I}(t)}
\end{aligned}
$$

from the formulars 17, 18, 19, 20, we can known that $\mathrm{PMV}_{I}$ and $\mathrm{PMV}_{Q}$ were directly related to in-phase and quadrature-phase disturbances respectively, and the symbol phase deviation can be represented by $\mathrm{PMV}_{I}+$ $j * \mathrm{PMV}_{Q}$, that was shown in Figure 2. The operation processing in $\mathrm{Y}$-polarization was same as $\mathrm{X}$-polarization.

\section{Simulation for $112 \mathrm{~Gb} / \mathrm{s}$ PDM-4QAM System}

The joint phase estimate and compensation simulation configuration for $112 \mathrm{~Gb} / \mathrm{s}$ PDM-4QAM system was shown in Figure 3. $2^{13}$ random bits were generated, and transmitting DSP tasks was shown in Figure 4(a) that were carried out at the Nyquist rate in MATLAB R2009b, the transmitting DSP was first performed pulse

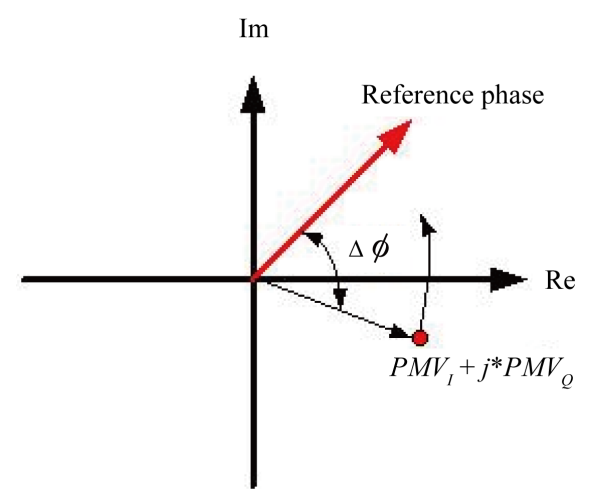

Figure 2. The sketch map of phase rotation based on $\mathrm{PMV}_{I}+j^{*} \mathrm{PMV}_{Q}$ coordinate. 

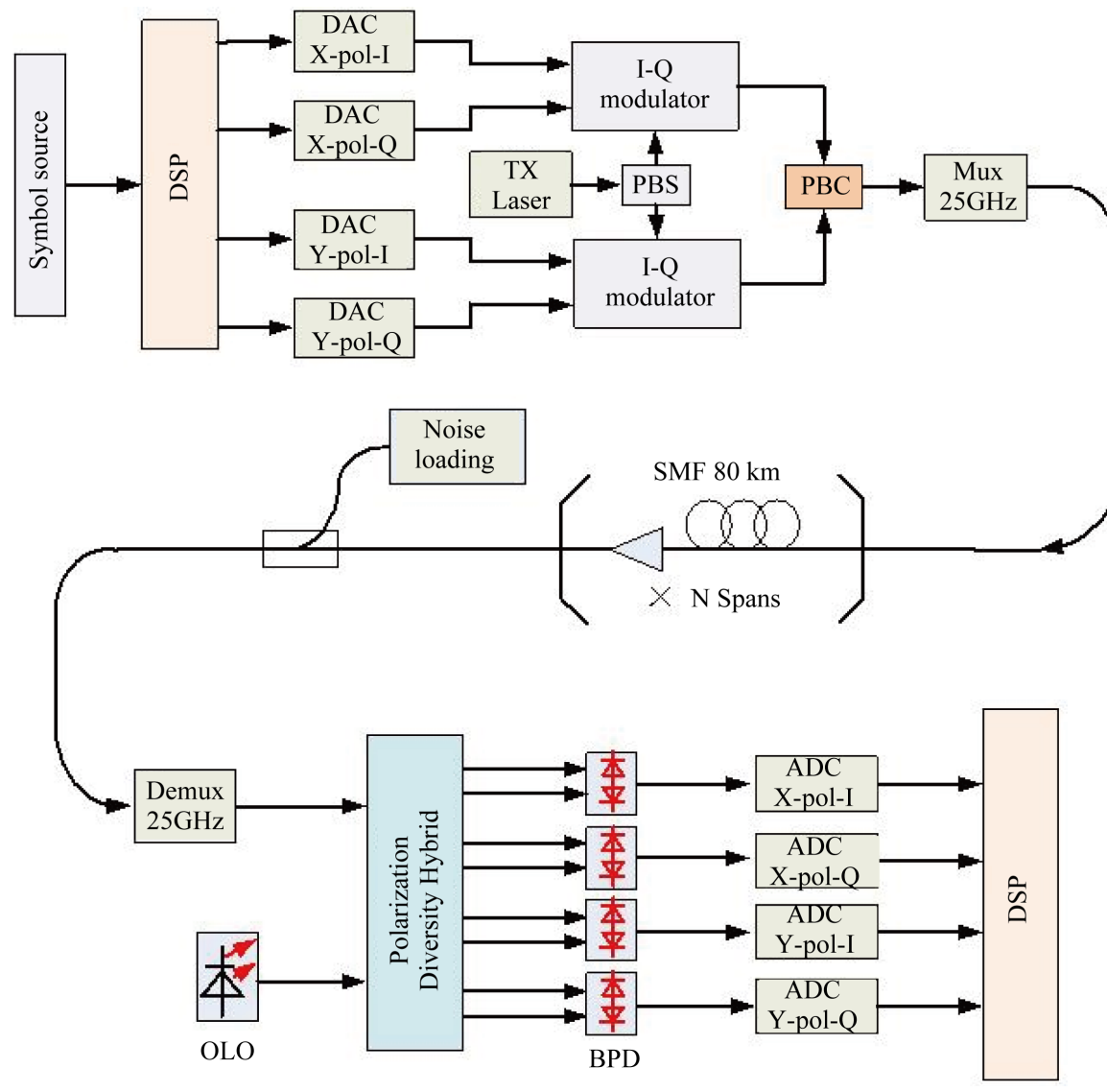

Figure 3. The experimental architecture of joint phase estimate and compensation for 112 Gb/s PDM-4QAM system.

shaping on transmitted data symbols, a root-raised cosine (RRC) pulse shape with a roff-off factor of 0.1 was assumed throughout all simulations. After, the four 28 Gbaud $I$ and $Q$ signals on both polarization were upsampled to 4 samples/symbol and launched into optisystem 9.0 to simulate the optical layer of the transmission system. Note that 4 samples/symbol are used in optisystem to provide enough simulation bandwidth, also, infinite DAC resolution was assumed. At the optical layer simulation, the TX and RX lasers were assumed to have a wavelength of $1554.12 \mathrm{~nm}$ and a linewidth of $100 \mathrm{KHz}$ respectively each, four ways RF pilots were loaded through two I-Q modulators which placed at X-pol and Y-pol respectively, and all the RF pilot with the frequency of $250 \mathrm{MHz}$. The power of the loaded RF pilot depends on the pilot-to-signal power ratio (PSPR), which was defined in [13], clearly, there was a trade-off between increasing and decreasing PSPR. The larger PSPR, the higher accuracy for phase deviation monitoring, but if PSPR exceeds a certain value, the pilot tone maybe disturbs data signals that lead to bit error rate sharply increase, if PSPR is too small, the pilot tone could be submerged by ASE noise and result in phase deviation monitoring is defeated, although that barely induces disturbance to data signals. So an optimized PSPR was carried out according to [6], and the PSPR was set at $-18 \mathrm{~dB}$. Finally, the hybrid modulating signal (contains PDM-4QAM signals and RF pilot tones) were respectively propagated over $10 \times 80 \mathrm{~km}, 20 \times 80 \mathrm{~km}, 30 \times 80 \mathrm{~km}$ of dispersion unmanaged standard singal mode fiber (SSMF) with attenuation of $\alpha=0.2 \mathrm{~dB} / \mathrm{km}$, dispersion $D=16.75 \mathrm{ps} /(\mathrm{nm} \cdot \mathrm{km})$, dispersion slope $S=0.075 \mathrm{ps} /\left(\mathrm{nm}^{2} \cdot \mathrm{km}\right)$, effective area $A=80 \mathrm{um}^{2}$, Kerr NL parameter $n_{2}=26 \times 10^{-21} \mathrm{~m}^{2} / \mathrm{W}$, and negligible PMD. An Erbium-doped fiber amplifier (EDFA) with a noise figure NF $=7 \mathrm{~dB}$ was placed at every span of $80 \mathrm{~km}$. Noise loading at the RX was carried out to sweep the received OSNR level. At the RX, the four signals out of the coherent front-end were launched into four balanced-photodetectors (BPDs) respectively, the output of each BPD was separated into two ways, one of was launched into high-pass filter to clear away RF pilot, after the signal was down sampled by ADC that processed in MATLAB. The other was launched into I-Q mixing unit, it's specific operation was shown in Figure 2. Eight channels signal from $I-Q$ mixing array were launched into 


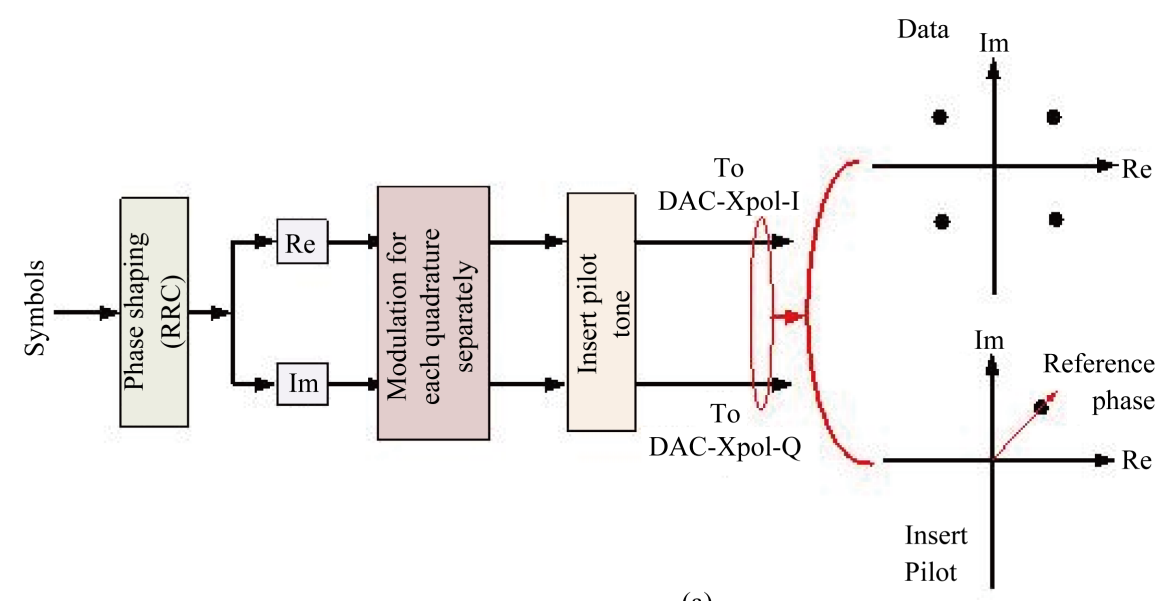

(a)

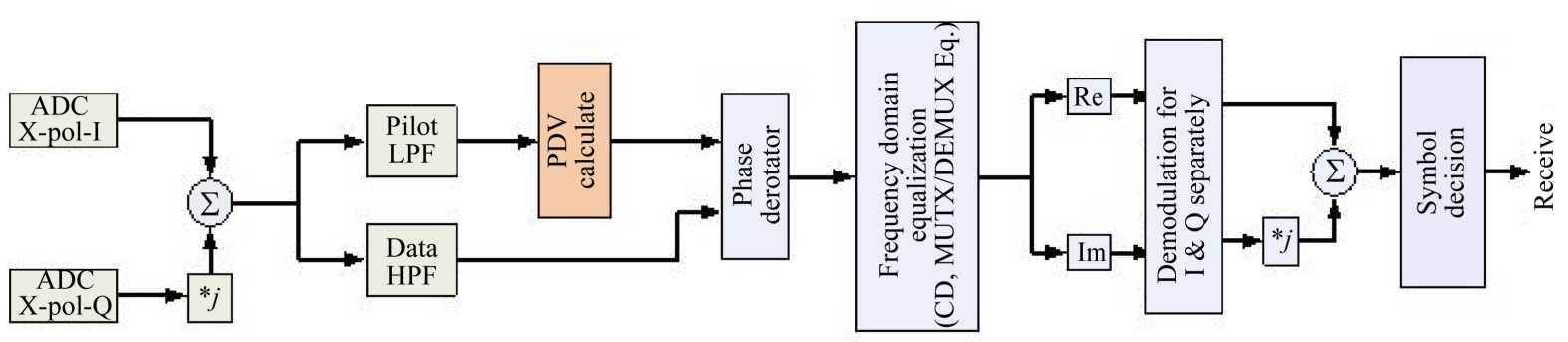

(b)

Figure 4. DSP tasks for transmission system in X-polarization: (a) TX side; (b) RX side.

eight LPFs respectively and to extract down-mixing signals, after the signal was downsampled by ADC that processed in MATLAB also. MATLAB where they were first downsampled to their Nyquist rate and then processed Figure 5(b). Finally, a bit-error rate (BER) level of $3 \times 10^{-3}$ was assumed in all simulations.

\section{Simulation Results and Discussion}

First, we performed PMV monitoring under conditions of 800 km, 1600 km, 2400 km SMF respectively, the launch powers were set at $0 \mathrm{dBm}$ for three simulations, and the OSNR level of receiver was maintained at 16.5 $\mathrm{dB}$ through a white light adjusted noise loading. In this paper we just provided the PMV monitored results corresponding to 128 symbols that to show monitoring feature in different nonlinear strength. And the monitored PMVs corresponding to three different distances were respectively showed in Figures 5-7. The fluctuation of PMV can stand for phase noise that created by the nonlinear effect in ultra long-haul fiber links. Through comparing, the fluctuation of PMVs under the SMF distance of $800 \mathrm{~km}$ was smooth compared with $1600 \mathrm{~km}$ and $2400 \mathrm{~km}$, that indicated the NL impairments was more serious along with the longer transmission distance.

Then, we performed phase noise compensation based on the proposed phase deviation monitoring technology, the operation specifics as follow: during each symbol cycle, we firstly monitored the PMVs mapping to $I$ and $Q$ phase in each polarizations, and calculate phase deviation for each symbol in each polarizations, after, the phase rotator performed phase rotating for each symbols according to the calculated phase deviation values to realize symbol constellation diagram phase correction, the receiving compensation process was shown in Figure 5, the CD impairments was compensated by the CD linear equalization with Viterbi and Viterbi phase estimation (VVPE) for FFCR [13]. To assess the transmission performance of 112 Gb/s PDM-4QAM system (both with and without the proposed NL phase compensation), the $Q$-factor was measured as a function of input power for several fixed transmission distances that were plotted in Figure 8. Figure 8(a) showed that the optimum launch power for all measured distances was approximately $-4 \mathrm{dBm}$, for the low input powers the main source of degradation was OSNR degradation due to accumulated ASE noise from the EDFAs in the fiber link, while for higher input powers the performance was degraded due to in-band nonlinearity effect. Applying the proposed 


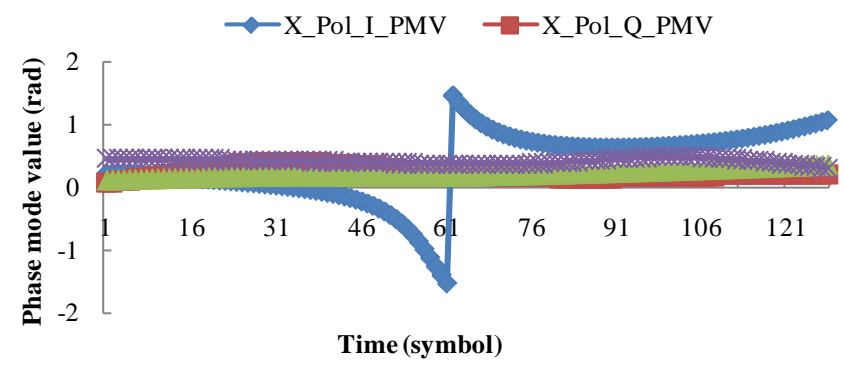

Figure 5. Monitored PMVs corresponding to 128 symbols (SMF: 800 km; OSNR: $16.5 \mathrm{~dB}$; Launch power: $0 \mathrm{dBm}$ ).

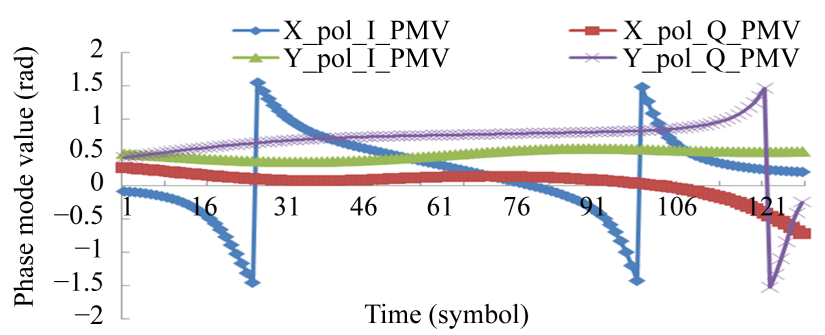

Figure 6. Monitored PMVs corresponding to 128 symbols (SMF: 1600 km; OSNR: $16.5 \mathrm{~dB}$; Launch power: $0 \mathrm{dBm}$ ).

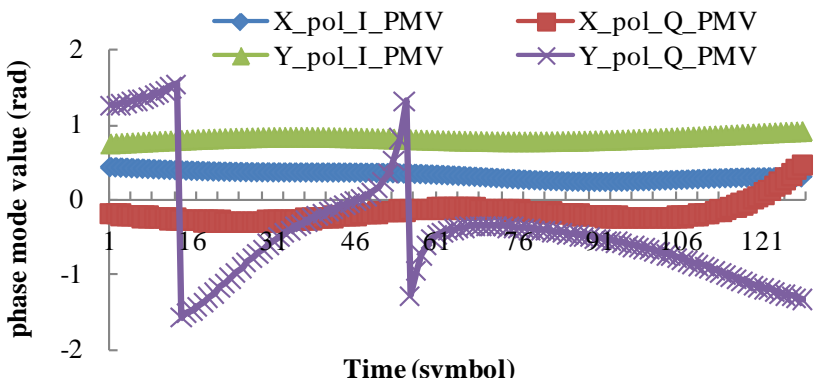

Time (symbol)

Figure 7. Monitored PMVs corresponding to 128 symbols (SMF: 2400 km; OSNR: $16.5 \mathrm{~dB}$; Launch power: $0 \mathrm{dBm}$ ).

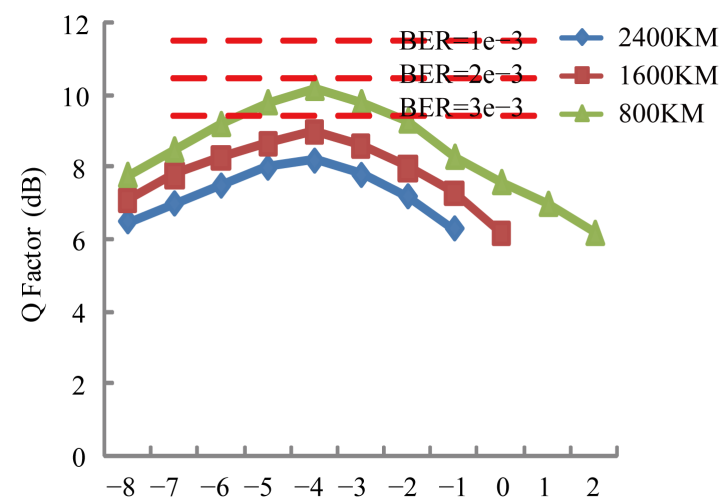

Launch Power (dBm)

(a)

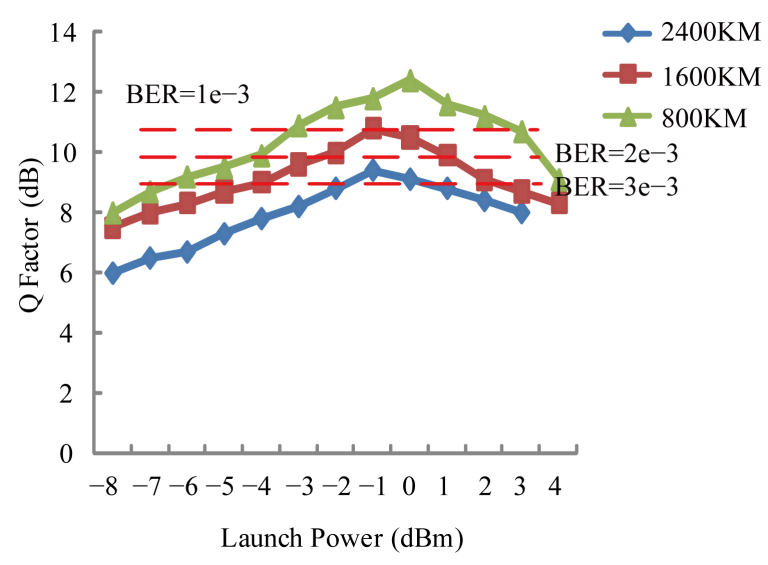

(b)

Figure 8. The system $Q$ values versus various launch power. (a) Without NL phase compensation; (b) With NL phase compensation. 
NL phase compensation that allowed for an increase in the optimum launch power by $3.5 \mathrm{~dB}$ for all measured distances, that was showed in Figure 8(b), it's also observed that the increase of input launch power margin from $5 \mathrm{~dB}$ to $10 \mathrm{~dB}$ at the distance of $1200 \mathrm{~km}$ with the FEC limit of $3 \times 10^{-3}$. Q-factor was plotted as a function of transmission distances at the optimum launch power (denoted as optimum $Q$-factor) was showed in Figure 9, it demonstrated that the proposed NLC yielded an improvement in optimum $Q$-factor by $2 \mathrm{~dB}$ at 1600 $\mathrm{km}$ and $1.5 \mathrm{~dB}$ at $2400 \mathrm{~km}$.

Constellation diagrams in condition of back to back and after $800 \mathrm{~km}, 1600 \mathrm{~km}$ and $2400 \mathrm{~km}$ transmission (both with and without NLC) were showed in Figure 10.

\section{Conclusion}

In this paper, we proposed a novel method of joint phase noise estimate (JPNE) for PDM-M-QAM $(M=4,16$,

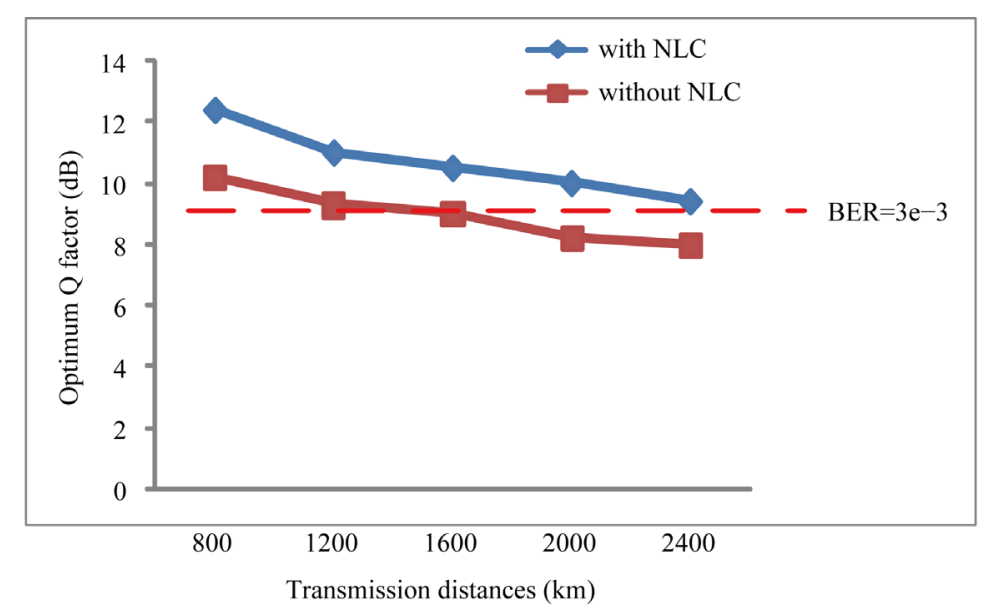

Figure 9. Performance comparison of transmission with and without NLC.

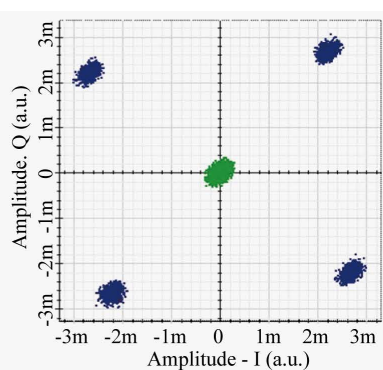

(a)

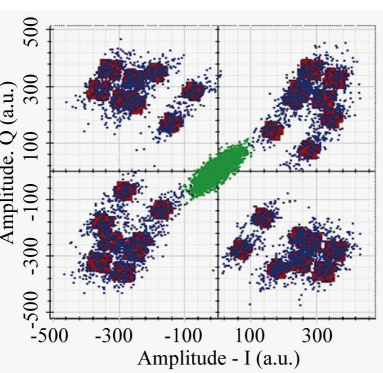

(b)

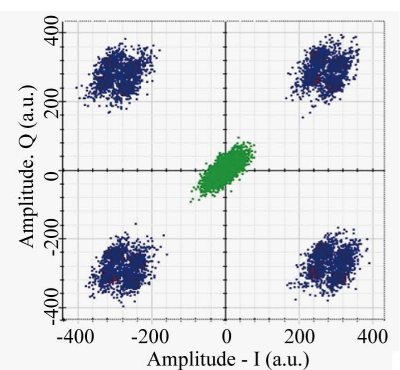

(c)

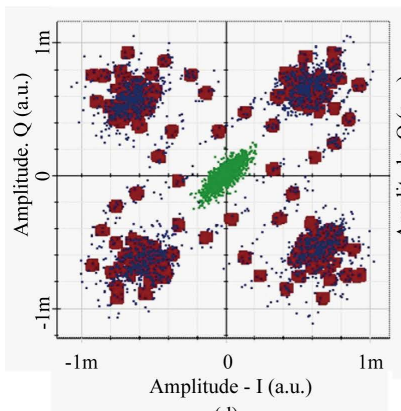

(d)

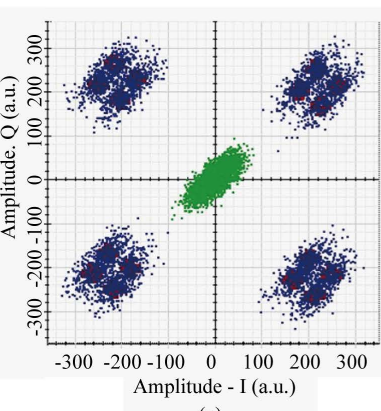

(e)

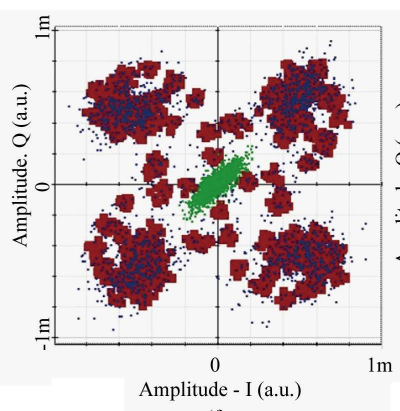

(f)

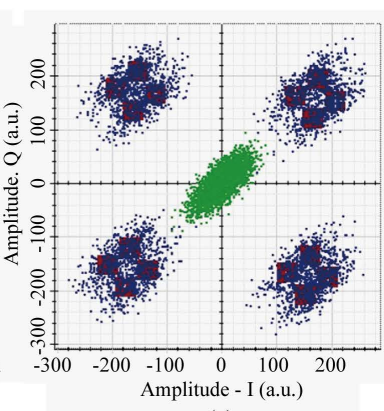

(g)

Figure 10. Simulation PDM-4QAM constellation diagrams at 112 Gbit/s. (a) Back to back without noise loading; (b) 800 km transmission without NLC at $-4 \mathrm{dBm}$; (c) $800 \mathrm{~km}$ transmission with NLC at $-4 \mathrm{dBm}$; (d) $1600 \mathrm{~km}$ transmission without NLC at $-1 \mathrm{dBm}$; (e) $1600 \mathrm{~km}$ transmission with NLC at $-1 \mathrm{dBm}$; (f) $2400 \mathrm{~km}$ transmission without NLC at $-1 \mathrm{dBm}$; (g) $2400 \mathrm{~km}$ transmission with NLC at $-1 \mathrm{dBm}$. 
$32,64, \ldots)$ transmission systems, and established the theoretical model to illustrate the operation mechanism. Through simulation, the random phase noise among four channels of $112 \mathrm{~Gb} / \mathrm{s}$ PDM-4QAM system was monitored; the results indicated that the phase perturbation increased with a long distance, meanwhile, the transmission performance for $112 \mathrm{~Gb} / \mathrm{s}$ PDM-4QAM system with the proposed NL compensation was investigated, over the range of distances examined. The optimum launch power was found to be $-4 \mathrm{dBm}$ with the linear compensation only. We also studied the maximum benefit available from the proposed NL compensation for single-channel transmission and found an increase in optimum launch power from $-4 \mathrm{dBm}$ to $0 \mathrm{dBm}$, with consequent increase in maximum reach by $50 \%$ to $2400 \mathrm{~km}$.

\section{Acknowledgements}

The work described in this paper was carried out with the support of the National Natural Science Foundation of China (61301226), and also supported by "the Fundamental Research Funds for the Central Universities", HUST: CXY13M001.

\section{References}

[1] Gnauck, A.H., Winzer, P.J., Konczykowska, A., Jorge, F., Dupuy, J., Riet, M., Charlet, G., Zhu, B. and Peckham, D.W. (2012) Generation and Transmission of 21.4-Gbaud PDM 64-QAM Using a Novel High-Power DAC Driving a Single I/Q Modulator. Journal of Lightwave Technology, 30, 532-536. http://dx.doi.org/10.1109/JLT.2011.2175200

[2] Fatadin, I., Ives, D. and Savory, S.J. (2009) Blind Equalization and Carrier Phase Recovery in a 16-QAM Optical Coherent System. Journal of Lightwave Technology, 27, 3042-3049. http://dx.doi.org/10.1109/JLT.2009.2021961

[3] Makovejs, S., Millar, D.S., Lavery, D., Behrens, C., Killey, R.I., Savory, S.J. and Bayvel, P. (2010) Characterization of Long-Haul 112 Gbit/s PDM-QAM-16 Transmission with and without Digital Nonlinearity Compensation. Optics Express, 18, 12939-12947. http://dx.doi.org/10.1364/OE.18.012939

[4] Khairuzzaman, Md., Zhang, C., Igarashi, K., Katoh, K. and Kikuchi, K. (2010) Equalization of Nonlinear Transmission impairments by Maximum-Likelihood-Sequence Estimation in Digital Coherent Receivers. Optics Express, 18, 4776.

[5] Morsy-Osman, M., Zhuge, Q., Chagnon, M., Xu, X. and Plant, D.V. (2013) Time Domain Hybrid QAM Based Rate-Adaptive Optical Transmissions Using High Speed DACs. Proc. of OFC, OTu3I.6, Los Angeles.

[6] Morsy-Osman, M., Chen, L.R. and Plant, D.V. (2011) Joint Mitigation of Laser Phase Noise and Fiber Nonlinearity for Polarization-Multiplexed QPSK and 16-QAM Coherent Transmission Systems. Proc. ECOC Tu.3.A.3.

[7] Lu, G.-W., Nakamura, M., Kamio, Y. and Miyazaki, T. (2007) Ultimate Linewidth-Tolerant 20-Gbps QPSK-Homodyne Transmission Using a Spectrum-Sliced ASE Light Source. Optics Express, 15, 7660.

[8] Morsy-Osman, M., Zhuge, Q., Chen, L.R. and Plant, D.V. (2011) Feedforward Carrier Recovery via Pilot-Aided Transmission for Single-Carrier Systems with Arbitrary M-QAM Constellations. Optics Express, 19, 24331.

[9] Ip, E. and Kahn, J.M. (2008) Coherent Detection in Optical Fiber Systems. Journal of Lightwave Technology, 26, 3416.

[10] Mateo, E.F., Zhou, X. and Li, G.F. (2011) Improved Digital Backward Propagation for the Compensation of InterChannel Nonlinear Effects in Polarization-Multiplexed WDM Systems. Optics Express, 19, 570.

[11] Tanimura, T., Nölle, M., Fischer, J.K. and Schubert, C. (2012) Analytical Results on Back Propagation Nonlinear Compensator with Coherent Detection. Optics Express, 20, 28779.

[12] Mateo, E.F., Yaman, F. and Li, G.F. (2010) Improved Digital Backward Propagation for the Compensation of InterChannel Nonlinear Effects in Polarization-Multiplexed WDM Systems. Optics Express, 18, 15144.

[13] Viterbi, A. (1983) Nonlinear Estimation of PSK-Modulated Carrier Phase with Application to Burst Digital Transmission. IEEE Transactions on Information Theory, 29, 543-551. http://dx.doi.org/10.1109/TIT.1983.1056713 
Scientific Research Publishing (SCIRP) is one of the largest Open Access journal publishers. It is currently publishing more than 200 open access, online, peer-reviewed journals covering a wide range of academic disciplines. SCIRP serves the worldwide academic communities and contributes to the progress and application of science with its publication.

Other selected journals from SCIRP are listed as below. Submit your manuscript to us via either submit@scirp.org or Online Submission Portal.
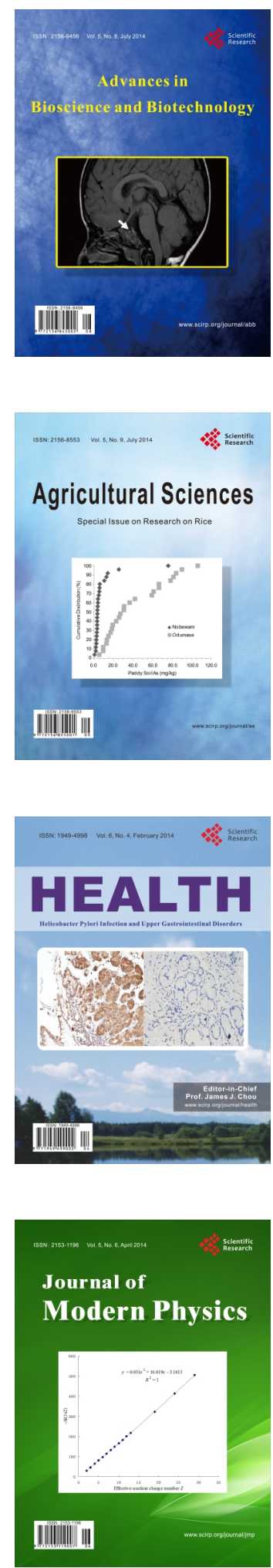
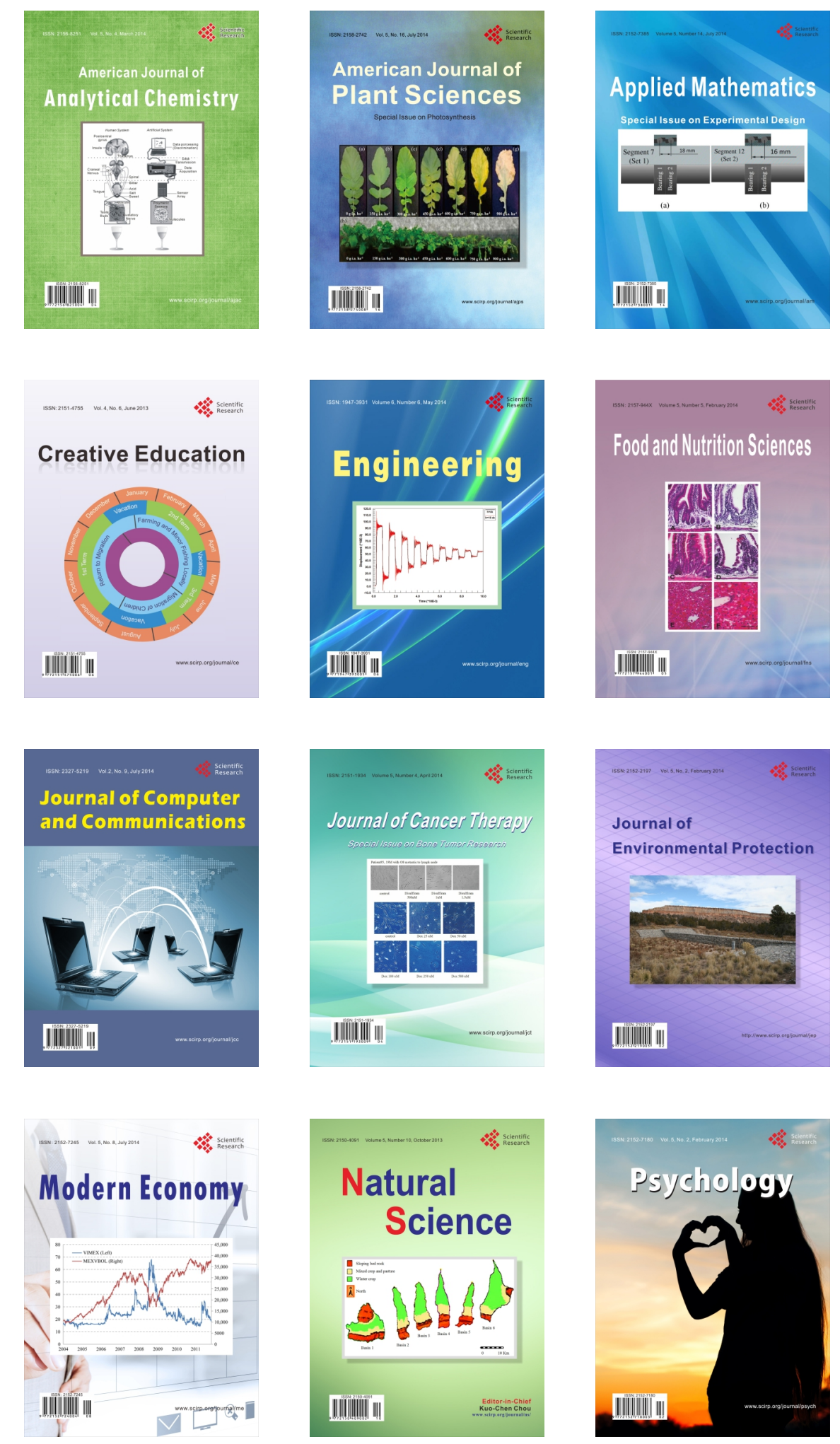